\title{
Warp signatures of the Galactic disk as seen in mid infrared from Midcourse Space Experiment
}

\begin{abstract}
S. Vig, S. K. Ghosh, and D. K. Ojha
Tata Institute of Fundamental Research, Homi Bhabha Road, Mumbai (Bombay) 400 005, India

e-mail: swarna@tifr.res.in

Received 24 January 2003 / Accepted 11 March 2005

Abstract. The gross features in the distribution of stars as well as warm $(T \gtrsim 100 \mathrm{~K})$ interstellar dust in the Galactic disk have been investigated using the recent mid infrared survey by Midcourse Space Experiment (MSX) at 8, 12, 14 and $21 \mu \mathrm{m}$ bands. An attempt has been made to determine the location of the Galactic mid-plane at various longitudes, using two approaches : (i) fitting exponential functions to the latitude profiles and (ii) statistical indicators. The former method is successful for the inner Galaxy $\left(-90^{\circ}<l \leq 90^{\circ}\right)$, and quantifies characteristic angular scales, $\gamma$, along latitude. These $\gamma \mathrm{s}$ have been translated to linear scale heights $\left(z_{\mathrm{h}}\right)$ and radial length scales $\left(R_{\mathrm{l}}\right)$ using geometric description of the Galactic disk. The distribution of warm dust in the Galactic disk is found to be characterised by $R_{\mathrm{l}}<6 \mathrm{kpc}$ and $60 \lesssim z_{\mathrm{h}} \lesssim 100 \mathrm{pc}$, consistent with other studies.

The location of the Galactic mid-plane as a function of longitude (in all 4 MSX bands), for stars as well as warm dust, has been searched for signatures of warp-like feature in their distribution, by fitting sinusoid with phase and amplitude as parameters. In every case, the warp signature has been detected. Carrying out an identical analysis of the DIRBE/COBE data (with lower angular resolution) in all its ten bands covering the entire infrared spectrum $(1.25-240 \mu \mathrm{m})$, also leads to detection of warp signatures with very similar phase as found from the MSX data. Our results have been compared with those from other studies.
\end{abstract}

Key words. Galaxy: disk - infrared: stars - infrared: ISM - ISM: dust, extinction

\section{Introduction}

The study of structural features of the Galactic disk is useful in understanding the spatial distribution of its constituents, viz. stars, interstellar gas and interstellar dust. Our Galaxy consists of the following kinematically distinct components: spheroidal (bulge, halo) and disk components (thin disk, thick disk). The disk component of the Galaxy has been studied extensively using several infrared surveys. Kent et al. (1991) obtained the three dimensional distribution of our Galaxy based on data at $2.4 \mu \mathrm{m}$ from the Spacelab IRT. Freudenreich (1996) used the Diffuse Infrared Background Experiment (DIRBE/COBE) data in $J, K, L$ and $M$ bands to model the Galactic disk.

One of the subtler features of the Galaxy is that of the warp structure, first detected through the $21 \mathrm{~cm}$ line emission of H I (Burke 1957; Kerr 1957). Various theories have been put forward to explain this phenomenon: gravitational interaction with a companion galaxy (Burke 1957; Kerr 1957; Hunter \& Toomre 1969; and others), effect of massive halo (Binney 1978, 1992), misaligned halo-disc axes leading to cosmic infall (Ostriker \& Binney 1989; Jiang \& Binney 1999), intergalactic magnetic fields (Battaner et al. 1990, 1991), dynamical friction between misaligned rotating halo and disc (Debattista \& Sellwood 1999), and more recently, intergalactic accretion flows onto the disc (Lopez-Corredoira et al. 2002a). Porcel et al. (1997) have explained the Galactic stellar warp using the tilted ring model of Rogstad et al. (1974). The warp in the stellar disk was detected from the distribution of sources in the Infrared Astronomical Satellite (IRAS) Point Source Catalog (Djorgovski \& Sosin 1989). Using the DIRBE data, Freudenreich et al. (1994), found evidence of warp for the stellar and the interstellar dust components of the Galaxy. The old stellar populations near the Galactic plane have been analysed for Galactic flare and warp from the Two Micron All Sky Survey (2MASS) by Lopez-Corredoira et al. (2002b).

Recently, the Midcourse Space Experiment (MSX) surveyed the entire Galactic plane in four mid infrared spectral bands $(8-21 \mu \mathrm{m})$ with high $\left(\sim 18^{\prime \prime}\right)$ angular resolution (Price et al. 2001). Taking advantage of the complete longitude coverage and superior angular resolution of this new dataset, we have attempted to extract information about structure of the Galactic disk and search for possible warp signature from the distribution of stellar as well as warm interstellar dust components of our Galaxy. For a direct and meaningful comparison, an identical analysis has also been carried out with the DIRBE data, albeit with poorer angular resolution.

In Sect. 2, we discuss the MSX and DIRBE datasets used for the present study. The data analysis procedures followed to extract structural features of the Galaxy and possible signatures of warp, have been described along with the results in Sect. 3. Based on a geometric description of the Galactic disk, a relation between the linear and the angular scale heights have 
been established in Sect. 4. Our results have been compared with others' in Sect. 5. In Sect. 6, a brief summary has been presented.

\section{Data sets}

The main objectives of the present study are to quantify parameters that characterise the structure of Galactic disk and search for possible signatures of warp from the distributions of stellar and warm interstellar dust components. The MSX data products have been used extensively. In addition, DIRBE data have also been used for comparison.

\subsection{Midcourse space experiment (MSX)}

The MSX carried out mid infrared observations of the Galactic plane $\left(|b| \leq 5^{\circ}\right)$ in four bands $A, C, D$ and $E$ with $\lambda(\Delta \lambda)$ corresponding to 8.28 (3.36), 12.13 (1.72), 14.65 (2.23), and 21.34 (6.24) $\mu \mathrm{m}$, respectively (Price et al. 2001). While the MSX Point Source Catalog, MSX PSC (Egan et al. 1999), has been used to study the distribution of point sources, the panoramic images in the four bands (pixel size $36^{\prime \prime} \times 36^{\prime \prime}$ ) have been used to study the diffuse emission. These images have already been corrected for the zodiacal emission using an updated version of CBZODY model described in Kennealy et al. (1993). The MSX PSC and the panoramic images were taken from IPAC (http://irsa.ipac.caltech.edu/applications/MSX/) and the MSX team.

We selected 4 samples of sources from the MSX PSC (one for each band), each based on the following criteria : (1) positive detection of the source in the particular band (quality flag $\geq 2$ ); (2) source located within the latitude range $|b| \leq 2.75^{\circ}$, in order to avoid regions with non-uniform sky coverages; and (3) sources brighter than the completeness limit of the catalogue for that band, viz. $0.158 \mathrm{Jy}, 1.58 \mathrm{Jy}, 1.58 \mathrm{Jy}$ and $3.16 \mathrm{Jy}$ for $8,12,14$ and $21 \mu \mathrm{m}$ bands, respectively (as determined from differential $\log N-\log S$ distributions). The completeness limit in A band corresponds to 6.42 magnitude at $8 \mu \mathrm{m}$, using the magnitude scale given by Egan et al. (1999). Our samples consist of $164723,25467,18239$ and 14254 sources in $8,12,14$ and $21 \mu \mathrm{m}$ bands, respectively. The $8 \mu \mathrm{m}$ band of MSX PSC being the most sensitive, the corresponding sample is the largest. Using MSX PSC data, Lumsden et al. (2002) have plotted known stars, planetary nebulae, H II regions and young stellar objects (YSOs) on colour-colour diagrams in Figs. 3-5 of their paper. We have used a colour cut of the form $\log _{10}\left(F_{14} / F_{12}\right)=0.87 \log _{10}\left(F_{21} / F_{8}\right)-0.61$ (equation of a straight line on a log-log plot; stars are located to the left of this line) which separates the H II regions, etc from the stars, as evident from their Fig. 3 (right panel). Using this colour cut we find the fraction of non-stellar objects to be $\sim 13 \%$. This is similar to IRAS Point Source Catalog, where $\sim 15 \%$ of the sources detected in both the mid infrared bands (12 and $25 \mu \mathrm{m})$ are non-stellar objects (Beichman et al. 1988).

\subsection{Emission from DIRBE sky survey}

The DIRBE instrument onboard COBE spacecraft carried out a full sky survey in 10 photometric bands centered at $1.25,2.2,3.5,4.9,12,25,60,100,140$ and $240 \mu \mathrm{m}$, albeit with limited angular resolution of $\sim 0.7^{\circ}$ (Boggess et al. 1992). We have used the Zodi-subtracted Mission Average (ZSMA) maps here, which were obtained from LAMBDA (http://lambda.gsfc.nasa.gov/product/cobe/dirbe_ products.cfm).

\section{Analysis and results}

\subsection{MSX Point Source Catalog (PSC) sources}

\subsubsection{Estimated distribution of stellar types and distance for our sample}

In order to estimate the distribution of stellar types of the mid infrared sources in our sample and their distances, we have used a modified version of the Besançon model of stellar population synthesis (A. Robin, private communication). An earlier version of the model has been described in Robin et al. (2003). The modified version of the model includes predictions of star counts in two mid infrared bands corresponding to the LW2 ( $7 \mu \mathrm{m})$ and LW3 $(15 \mu \mathrm{m})$ filters of ISOCAM, which are crucial for the present study. This model synthesizes stars belonging to the populations of the thin disk, the thick disk, the stellar halo and the outer bulge including the warp and flare features. This model has also been used to estimate the contribution of all stars, including those not detected by MSX PSC, to the total mid IR emission (see Sect. 3.2.1). Simulations have been carried out over $10 \times 10 \mathrm{deg}^{2}$ area for $|b|<5^{\circ}$ and $30^{\circ}<l \leq 330^{\circ}$ on a grid with $30^{\circ}$ spacing. The simulations covered distances upto $20 \mathrm{kpc}$ from the Sun and stars upto apparent magnitude of 14.0 at $7 \mu \mathrm{m}$. The extinction due to interstellar dust has been neglected in the model simulations.

As mentioned earlier, the completeness limit of MSX PSC for A band $(8 \mu \mathrm{m})$ corresponds to $6.4 \mathrm{mag}$ at $8 \mu \mathrm{m}$, [8]. This translates to a value of $6.5 \mathrm{mag}$ at $7 \mu \mathrm{m}$, [7], for the stellar types in our sample (the mean difference of the ([7]-[8]) colour, is $\sim 0.1 \mathrm{mag}$; for various effective temperatures covered in our sample, as evident below). Accordingly, we have considered the predicted star counts from the simulations with [7] $<6.5$, to be representative of our MSX PSC sample.

The mean Galactocentric distance for our sample, is found to vary from $\sim 4 \mathrm{kpc}$ towards the Galactic centre $\left(l \sim 0^{\circ}\right)$ to $\sim 11 \mathrm{kpc}$ towards the anti-centre direction $\left(l \sim 180^{\circ}\right)$. Most of the sources are dominated by $\mathrm{K}$ and $\mathrm{M}$ giants $(31 \% \mathrm{~K}$ and $59 \% \mathrm{M}$ giants). The rest are AGB stars $(6 \%)$ and earlier than G dwarfs (4\%).

\subsubsection{Latitude distribution of stellar sources and warp signature}

The structure of the Galactic disk has been studied using the local distribution of stellar sources of our sample by constructing latitude profiles (LP) of stars for 36 longitude bins, each $10^{\circ}$ wide. The bin size along latitude is $0.1^{\circ}$. The stellar density 
distribution in the Galaxy is generally represented by an exponential function of the form $n(z, R) \propto \mathrm{e}^{-\frac{z}{z_{\mathrm{h}}}-\frac{R}{R_{1}}}$, where $z$ is the height above the Galactic plane, $R$ is the Galactocentric distance, $z_{\mathrm{h}}$ and $R_{1}$ represent the characteristic scale height and radial scale length, respectively. However, all relevant measurements are (approximately) heliocentric, and conveniently presented in Galactic coordinates $(l, b)$. Later (Sect. 4 ), we shall relate the linear and the angular scales using a geometric description of the Galactic disk and show that exponential functions provide good fits to the latitude profiles too. Let $N(b)$ be the observed latitude profile representing the number of stars as a function of latitude, $b$, for a given longitude zone. At first, we explore both the exponential and Gaussian functions for fitting $N(b)$, as:

$N(b)=\alpha_{\mathrm{e}} \mathrm{e}^{-\frac{\left|b-\beta_{\mathrm{e}}\right|}{\gamma_{\mathrm{e}}}}+\delta_{\mathrm{e}}+\epsilon_{\mathrm{e}} b$

$N(b)=\alpha_{\mathrm{g}} \mathrm{e}^{-\left(\frac{b-\beta \mathrm{g}}{\gamma_{\mathrm{g}}}\right)^{2}}+\delta_{\mathrm{g}}+\epsilon_{\mathrm{g}} b$

where $\alpha, \beta, \gamma, \delta$ and $\epsilon$ are the free parameters. The subscripts e and $\mathrm{g}$ refer to the exponential and Gaussian functions, respectively. The parameters $\alpha_{\mathrm{e}}$ and $\alpha_{\mathrm{g}}$ represent the amplitude; $\beta_{\mathrm{e}}$ and $\beta_{\mathrm{g}}$ represent the position of the Galactic midplane defined by the MSX PSC stars; $\gamma_{\mathrm{e}}$ and $\gamma_{\mathrm{g}}$ represent the angular scale height; $\delta_{\mathrm{e}}, \delta_{\mathrm{g}}$ and $\epsilon_{\mathrm{e}}, \epsilon_{\mathrm{g}}$ represent the background, if any, limited to linear dependence on latitude. Generally, $\beta=0$ is expected if the peak of the latitude profile appears at $b=0$. Any non-zero value signifies a shift. The fittings have been carried out for all the 36 longitude bins using a non-linear least squares method. For each of the four wavebands, good fits were obtained for the inner half of the Galactic plane towards the Galactic center i.e. $-90^{\circ}<l \leq 90^{\circ}$ and very poor fits for the outer half, i.e. towards the Galactic anti-center $90^{\circ}<l \leq 270^{\circ}$. Predictions of the modified Besançon model also show the same trend for the distribution of stars ([7] $<6.5 \mathrm{mag})$. Figure 1 shows the latitude profiles (MSX PSC, A band) for three representative longitude bins $\left(30^{\circ}, 60^{\circ}\right.$ and $\left.180^{\circ}\right)$, including functional fits for the two longitudes in the inner Galaxy. For all the four bands, the exponential function fits the latitude profiles better than the Gaussian function, which is consistent with our understanding of the Galactic disk. Consequently, the results pertaining to the exponential function only have been presented here. Table 1 lists the mean value and standard deviation of the parameter $\gamma$ for each waveband, as obtained from the 18 longitude bins covering the inner Galactic plane.

Next we explore possible signatures of warping in the Galactic disk. To quantify any evidence for the Galactic warp from the local distribution of stars, we search for systematic variation of $\beta$ s with longitude. Djorgovski \& Sosin (1989), used a sinusoid function to fit the distribution of IRAS point sources. A similar approach has been adopted here, but without the additive constant term, thereby assuming that the Sun is located at the local midplane. Thus, the function used to fit the longitude variation of the profile peak is:

$\beta(l)=\beta_{0} \times \sin (l-\phi)$

where $\beta$ represents the best fit to $\beta_{\mathrm{e}}(l)$ of Eq. (1) obtained for $-90^{\circ}<l \leq 90^{\circ}$. Although useful, this is admittedly an
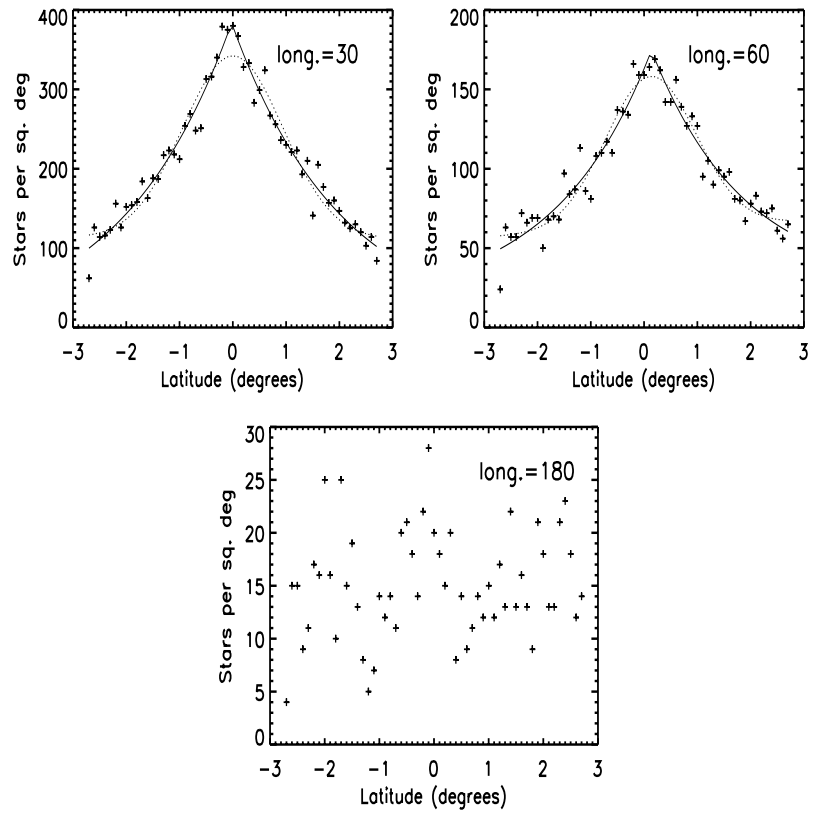

Fig. 1. The number density of stars as a function of latitude (latitude profile) detected in the $A$ band of MSX PSC for the longitude bins $30^{\circ}$, $60^{\circ}$ and $180^{\circ}$. The solid and the dotted lines represent best fit exponential and Gaussian functions, respectively (for $l=30^{\circ}$ and $60^{\circ}$ ).

Table 1. Characteristic angular scale, $\gamma$, of latitude profiles obtained by fitting exponentials to the MSX PSC sample, MSX emission and DIRBE emission for the 18 longitude bins covering $-90^{\circ}<l \leq 90^{\circ}$.

\begin{tabular}{ccc}
\hline \hline $\begin{array}{c}\text { Wavelength } \\
\mu \mathrm{m} \text { (band) }\end{array}$ & \multicolumn{2}{c}{$\begin{array}{c}\text { Exponential } \\
\mathrm{e}_{\mathrm{e}} \text { in deg(s.d.) }\end{array}$} \\
\hline & MSX PSC & MSX Diffuse emission \\
\hline $8.3(\mathrm{~A})$ & $1.29(0.67)$ & $1.69(0.45)$ \\
$12.1(\mathrm{C})$ & $1.27(0.57)$ & $0.92(0.41)$ \\
$14.7(\mathrm{D})$ & $1.25(0.70)$ & $0.71(0.22)$ \\
$21.3(\mathrm{E})$ & $1.04(0.96)$ & $0.56(0.28)$ \\
\hline & & DIRBE emission \\
\hline 1.25 & & $1.94(1.18)$ \\
2.2 & & $2.74(0.78)$ \\
3.5 & & $1.93(0.54)$ \\
4.9 & & $1.57(0.47)$ \\
12 & & $1.29(0.67)$ \\
25 & & $1.00(0.46)$ \\
60 & & $0.92(0.33)$ \\
100 & & $1.23(0.77)$ \\
140 & & $1.19(0.69)$ \\
240 & & $1.30(0.87)$ \\
\hline
\end{tabular}

approximate approach due to the fact that the measurements are heliocentric (hence, lines of sight along different longitudes cover different galactocentric distances of the disc), whereas a true sinusoid due to any warp feature is expected only for a galactocentric observer. We crudely relate the parameters $\beta$ 。 and $\phi$ to quantify the local warp signature (hereafter WS) indicating amplitude and phase respectively. This phase, $\phi$, corresponds to the position angle (longitude) of the line of intersection between the $b=0$ plane and the warped Galactic plane. In order to carry out a quantitative comparison with a "null" hypothesis (i.e. WS absent), we also fit a constant function, 
Table 2. The values of the best fit parameters of the warp signature, WS (sinusoid with amplitude $\beta_{\circ}$ and phase $\phi$ ) to the longitude variation $\left(-90^{\circ}<l \leq 90^{\circ}\right)$ of locations of the peaks of latitude profile, LP, obtained from the exponential fitting to: the MSX PSC sources $\left(|b| \leq 2.75^{\circ}\right)$, diffuse emission in MSX bands $\left(|b| \leq 5^{\circ}\right)$, and emission in DIRBE bands $\left(|b| \leq 5^{\circ}\right)$. The free parameter, $k$, for the null hypothesis is also tabulated. The ratio of $\chi^{2}$ per degree of freedom, $R$, for the best fitting null case and the WS case, is also presented. A value of $R>1$ indicates that the sinusoid is a better fit.

\begin{tabular}{ccccc}
\hline \hline $\begin{array}{c}\lambda \\
(\mu \mathrm{m})\end{array}$ & $\begin{array}{c}\beta_{\circ} \\
(\mathrm{deg})\end{array}$ & $\begin{array}{c}\phi \\
(\mathrm{deg})\end{array}$ & $\begin{array}{c}k \\
(\mathrm{deg})\end{array}$ & $R$ \\
\hline \multicolumn{5}{c}{ MSX PSC } \\
\hline 8.3 & $0.44 \pm 0.11$ & $3.1 \pm 14$ & $-0.04 \pm 0.10$ & 1.95 \\
12.1 & $0.39 \pm 0.10$ & $3.5 \pm 16$ & $-0.00 \pm 0.10$ & 1.80 \\
14.7 & $0.47 \pm 0.15$ & $3.2 \pm 19$ & $-0.04 \pm 0.13$ & 1.53 \\
21.3 & $0.42 \pm 0.13$ & $6.7 \pm 18$ & $-0.10 \pm 0.11$ & 1.53 \\
\hline \multicolumn{5}{c}{ MSX diffuse emission } \\
\hline 8.3 & $0.40 \pm 0.10$ & $18 \pm 14$ & $-0.13 \pm 0.09$ & 1.81 \\
12.1 & $0.57 \pm 0.16$ & $11 \pm 17$ & $-0.15 \pm 0.14$ & 1.58 \\
14.7 & $0.44 \pm 0.14$ & $6.2 \pm 18$ & $-0.13 \pm 0.12$ & 1.60 \\
21.3 & $0.69 \pm 0.19$ & $-6.3 \pm 16$ & $0.07 \pm 0.17$ & 1.75 \\
\hline \multicolumn{5}{c}{ DIRBE emission } \\
\hline 1.25 & $1.33 \pm 0.37$ & $72 \pm 16$ & $-0.71 \pm 0.29$ & 1.30 \\
2.2 & $0.51 \pm 0.08$ & $19 \pm 9.5$ & $-0.08 \pm 0.10$ & 3.11 \\
3.5 & $0.38 \pm 0.06$ & $15 \pm 9.4$ & $-0.04 \pm 0.07$ & 3.20 \\
4.9 & $0.34 \pm 0.07$ & $15 \pm 11$ & $-0.04 \pm 0.07$ & 2.63 \\
12 & $0.36 \pm 0.11$ & $18 \pm 17$ & $0.12 \pm 0.09$ & 1.50 \\
25 & $0.39 \pm 0.16$ & $11 \pm 23$ & $-0.06 \pm 0.12$ & 1.30 \\
60 & $0.45 \pm 0.15$ & $12 \pm 19$ & $-0.07 \pm 0.12$ & 1.46 \\
100 & $0.40 \pm 0.11$ & $13 \pm 16$ & $-0.10 \pm 0.10$ & 1.65 \\
140 & $0.37 \pm 0.11$ & $16 \pm 17$ & $-0.12 \pm 0.09$ & 1.52 \\
240 & $0.37 \pm 0.11$ & $16 \pm 17$ & $-0.12 \pm 0.09$ & 1.48 \\
\hline \multicolumn{5}{c}{}
\end{tabular}

$\beta(l)=k$, to the same data sets. The resulting best fit parameters along with their standard errors have been presented in Table 2. The data and the fitted functions are displayed in Fig. 2. The WS has been clearly detected in all the 4 bands as is evident from the ratio of $\chi^{2}$ s per degree of freedom $(R>1)$. On repeating our analysis with a larger sample of MSX PSC sources with latitude cut relaxed to $|b|<5^{\circ}$, again we detect WS with very similar parameters.

Since only the inner half of the Galactic plane could be studied using the above approach, we pursue additional measures of statistical nature to quantify the location of the Galactic midplane, which could be effective for the entire longitude range. We have explored two statistical measures of central tendency, viz., the mean and the median latitude. The mean refers to the average latitude of the MSX PSC sources of our sample for a particular longitude bin. The median latitude refers to a value such that the total number of stars located at higher and lower latitudes are equal. The mean is a good measure of central tendency for roughly symmetric distributions but can be misleading for skewed distributions. The median should be more informative for such distributions.

The mean and median latitudes for different Galactic longitude bins have been displayed as histograms in Fig. 3. A systematic deviation from the nominal Galactic plane $(b=0)$ is clearly visible in this figure. Both the statistical indicators (SI)
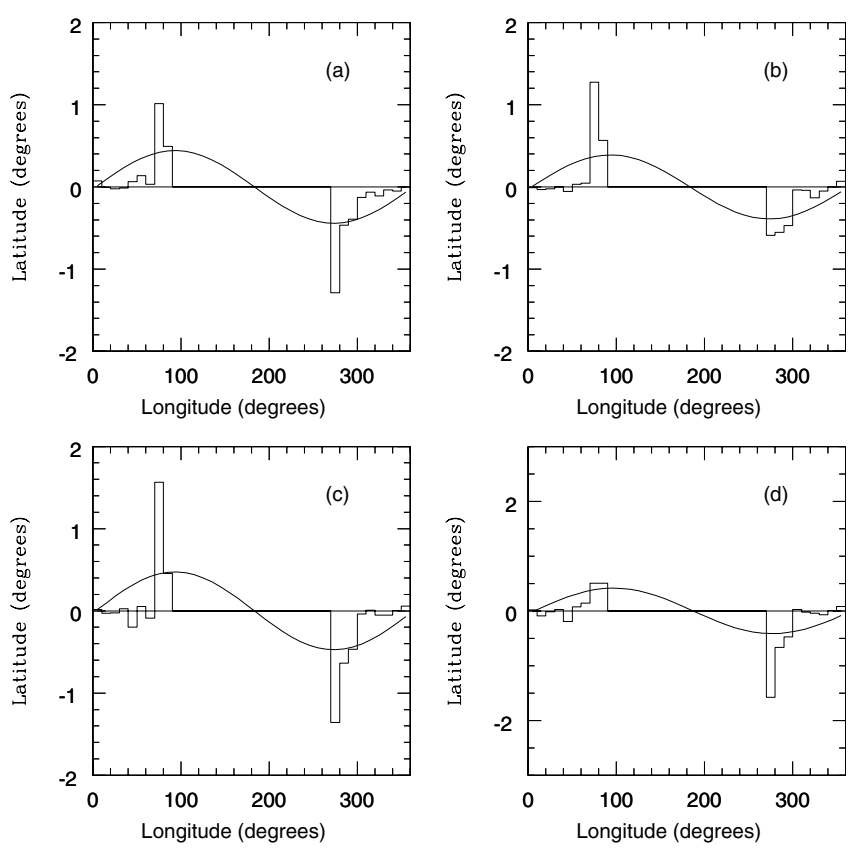

Fig. 2. The variation of the location of Galactic midplane with longitude, $\beta(l)$, as obtained from fitting exponential to the latitude profiles (LP) for our MSX PSC sample (histogram) for the inner Galaxy in 4 MSX bands. The solid lines refer to the extracted WS in a) $8 \mu \mathrm{m}$, b) $12 \mu \mathrm{m}$, c) $14 \mu \mathrm{m}$, and d) $21 \mu \mathrm{m}$ bands.
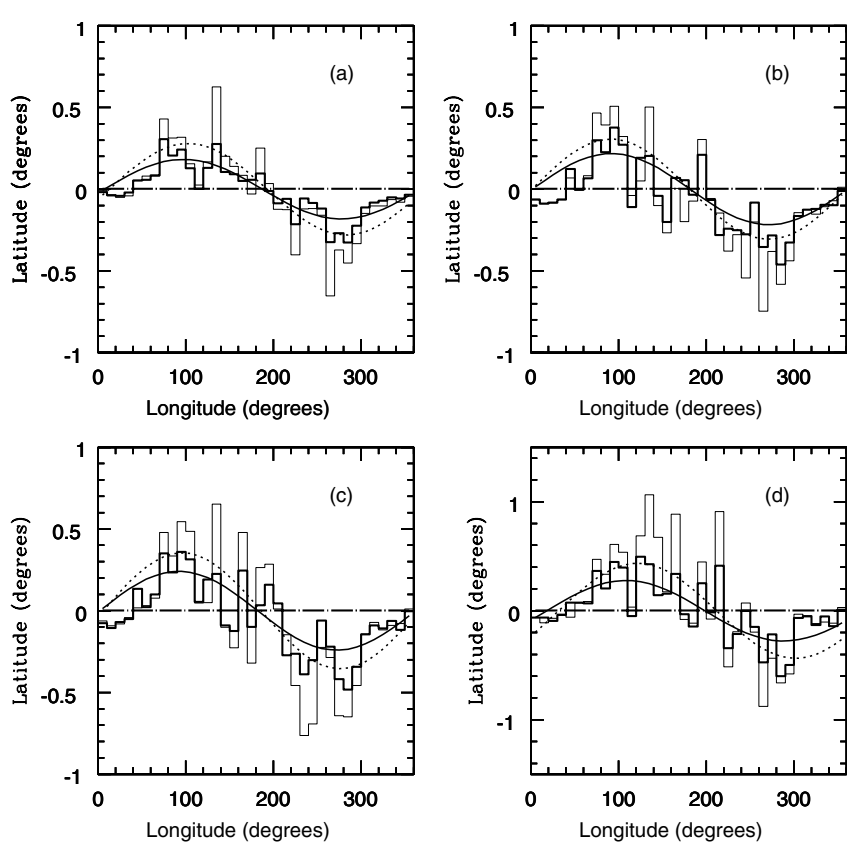

Fig. 3. The variation of the SIs of the Galactic midplane, viz. mean (thick line histogram) and median (thin line histogram) latitude as a function of longitude for our MSX PSC sample in the 4 MSX bands: a) $8 \mu \mathrm{m}$, b) $12 \mu \mathrm{m}$, c) $14 \mu \mathrm{m}$, and d) $21 \mu \mathrm{m}$. The solid and the dotted curves represent the extracted WS from the mean and median latitudes, respectively.

are positive over one half of the longitude range $\sim 0^{\circ}-190^{\circ}$ and negative in the other half. The positive and the negative peaks occur at $l \sim 90^{\circ}-100^{\circ}$ and $\sim 260^{\circ}-280^{\circ}$, respectively. This is the general trend for all the 4 bands of MSX. Similar to the 
Table 3. The values of the best fit parameters of the WS (sinusoid with amplitude $b_{\circ}$ and phase $\left.\phi\right)$ to the longitude variation $\left(0^{\circ}<l \leq 360^{\circ}\right)$ of the statistical indicators SI of Galactic midplane, viz., the mean \& median for: MSX PSC stars $\left(|b| \leq 2.75^{\circ}\right)$, intensity weighted \& median latitude of emission for MSX and DIRBE bands $\left(|b| \leq 5^{\circ}\right)$. The free parameter, $k$, for the null hypothesis is also tabulated. The ratio of $\chi^{2}$ per degree of freedom, $R$, for the best fitting null case and the WS case, is also presented $(R>1$ indicates the sinusoid to be a better fit).

\begin{tabular}{|c|c|c|c|c|}
\hline $\begin{array}{c}\lambda \\
\mu \mathrm{m}\end{array}$ & $\begin{array}{c}b_{\circ} \\
(\mathrm{deg})\end{array}$ & $\begin{array}{c}\phi \\
(\mathrm{deg})\end{array}$ & $\begin{array}{c}k \\
(\mathrm{deg})\end{array}$ & $\bar{R}$ \\
\hline \multicolumn{5}{|c|}{ MSX PSC: Mean, Median } \\
\hline \multirow[t]{2}{*}{8.3} & $0.18 \pm 0.02$ & $7.3 \pm 5.9$ & $-0.01 \pm 0.02$ & 3.75 \\
\hline & $0.28 \pm 0.03$ & $13 \pm 7.3$ & $-0.02 \pm 0.04$ & 2.78 \\
\hline \multirow[t]{2}{*}{12.1} & $0.22 \pm 0.03$ & $1.3 \pm 8.5$ & $-0.05 \pm 0.03$ & 2.15 \\
\hline & $0.31 \pm 0.05$ & $1.1 \pm 9.2$ & $-0.07 \pm 0.05$ & 1.98 \\
\hline \multirow[t]{2}{*}{14.7} & $0.24 \pm 0.03$ & $1.9 \pm 7.8$ & $-0.04 \pm 0.04$ & 2.46 \\
\hline & $0.35 \pm 0.06$ & $6.0 \pm 9.7$ & $-0.05 \pm 0.06$ & 1.95 \\
\hline \multirow[t]{2}{*}{21.3} & $0.28 \pm 0.04$ & $18 \pm 9.0$ & $0.01 \pm 0.04$ & 2.16 \\
\hline & $0.43 \pm 0.08$ & $32 \pm 10$ & $0.07 \pm 0.07$ & 1.83 \\
\hline \multicolumn{5}{|c|}{ MSX Diffuse emission: Intensity weighted, Median } \\
\hline \multirow[t]{2}{*}{8.3} & $0.35 \pm 0.05$ & $1.8 \pm 8.9$ & $0.02 \pm 0.06$ & 2.19 \\
\hline & $0.19 \pm 0.04$ & $-2.7 \pm 13$ & $0.11 \pm 0.03$ & 1.18 \\
\hline \multirow[t]{2}{*}{12.1} & $0.43 \pm 0.06$ & $-15 \pm 7.6$ & $0.01 \pm 0.06$ & 2.64 \\
\hline & $0.38 \pm 0.06$ & $-23 \pm 8.3$ & $0.06 \pm 0.06$ & 2.30 \\
\hline \multirow[t]{2}{*}{14.7} & $0.58 \pm 0.10$ & $-12 \pm 9.6$ & $-0.03 \pm 0.10$ & 2.01 \\
\hline & $0.99 \pm 0.22$ & $-25 \pm 13$ & $-0.16 \pm 0.19$ & 1.51 \\
\hline \multirow[t]{2}{*}{21.3} & $0.51 \pm 0.09$ & $-14 \pm 10$ & $0.08 \pm 0.08$ & 1.82 \\
\hline & $0.54 \pm 0.08$ & $-25 \pm 8.7$ & $0.05 \pm 0.08$ & 2.22 \\
\hline \multicolumn{5}{|c|}{ DIRBE emission : Intensity weighted, Median } \\
\hline \multirow[t]{2}{*}{1.25} & $0.22 \pm 0.07$ & $68 \pm 17$ & $-0.08 \pm 0.05$ & 1.18 \\
\hline & $0.39 \pm 0.11$ & $65 \pm 17$ & $-0.24 \pm 0.08$ & 1.05 \\
\hline \multirow[t]{2}{*}{2.2} & $0.20 \pm 0.05$ & $34 \pm 14$ & $-0.04 \pm 0.04$ & 1.41 \\
\hline & $0.30 \pm 0.08$ & $30 \pm 15$ & $-0.14 \pm 0.06$ & 1.20 \\
\hline \multirow[t]{2}{*}{3.5} & $0.26 \pm 0.04$ & $15 \pm 9.8$ & $-0.03 \pm 0.04$ & 1.95 \\
\hline & $0.37 \pm 0.07$ & $11 \pm 11$ & $-0.14 \pm 0.06$ & 1.55 \\
\hline \multirow{2}{*}{4.9} & $0.30 \pm 0.04$ & $6.8 \pm 8.6$ & $-0.03 \pm 0.05$ & 2.27 \\
\hline & $0.41 \pm 0.07$ & $3.8 \pm 9.3$ & $-0.14 \pm 0.06$ & 1.80 \\
\hline \multirow[t]{2}{*}{12} & $0.39 \pm 0.05$ & $-13 \pm 7.0$ & $-0.04 \pm 0.06$ & 2.89 \\
\hline & $0.51 \pm 0.08$ & $-11 \pm 8.9$ & $-0.19 \pm 0.08$ & 1.85 \\
\hline \multirow[t]{2}{*}{25} & $0.35 \pm 0.04$ & $-6.8 \pm 7.3$ & $-0.01 \pm 0.05$ & 2.79 \\
\hline & $0.49 \pm 0.08$ & $-3.8 \pm 9.3$ & $-0.16 \pm 0.07$ & 1.86 \\
\hline \multirow[t]{2}{*}{60} & $0.54 \pm 0.07$ & $-3.8 \pm 7.1$ & $-0.03 \pm 0.08$ & 2.89 \\
\hline & $0.68 \pm 0.11$ & $-6.1 \pm 9.6$ & $0.18 \pm 0.11$ & 1.87 \\
\hline \multirow[t]{2}{*}{100} & $0.49 \pm 0.06$ & $-8.2 \pm 7.0$ & $-0.04 \pm 0.07$ & 2.89 \\
\hline & $0.64 \pm 0.09$ & $-9.1 \pm 9.0$ & $-0.19 \pm 0.10$ & 1.95 \\
\hline \multirow[t]{2}{*}{140} & $0.52 \pm 0.06$ & $-11 \pm 7.2$ & $-0.05 \pm 0.08$ & 2.78 \\
\hline & $0.65 \pm 0.10$ & $-11 \pm 8.9$ & $-0.20 \pm 0.10$ & 1.95 \\
\hline \multirow[t]{2}{*}{240} & $0.54 \pm 0.07$ & $-13 \pm 7.4$ & $-0.04 \pm 0.08$ & 2.72 \\
\hline & $0.65 \pm 0.10$ & $-11 \pm 8.9$ & $-0.19 \pm 0.10$ & 1.97 \\
\hline
\end{tabular}

case of $\beta$ above for the exponential fits (Eq. (3)), we fit a sinusoid (with $b_{\circ}$ and $\phi$ as parameters) to the mean and the median latitudes giving equal weightage to each longitude bin. Once again, the "null" hypothesis is also explored for comparison. The resulting best fit parameters along with standard errors, for all the cases have been presented in Table 3. The corresponding fitted functions for respective bands have been overplotted in Fig. 3. The signature of warp is clearly indicated from both the statistical indicators (SIs) for all the 4 MSX bands. This is also evident from the ratio of the $\chi^{2} \mathrm{~s}(R>1)$. These findings are statistically further strengthened by the fact that the values of the phase, $\phi$, are consistent (within errors) among all four bands of MSX and also matches that expected from the known features of the Galactic stellar warp (Djorgovski \& Sosin 1989; see Sect. 5.2.1). The SI approach seems to be more successful than LP. This could be because the statistical determinations of central tendencies are inherently more immune to fluctuations than procedures involving fitting of a specific function.

The amplitude of the sinusoid is lower for the SI than the $\beta \mathrm{s}$ obtained from the fitting of the latitude profile. Among the mean and median, the displacement is larger for the latter case than the former. The mean provides a better quality fit to sinusoid than the median, based on comparison of corresponding reduced $\chi^{2} \mathrm{~s}$.

\subsection{Diffuse emission from MSX images}

Diffuse mid infrared emission is expected from the warmer $(T \gtrsim 100 \mathrm{~K})$ interstellar dust heated by nearby luminous stars. The emission in the mid infrared (obtained from the MSX panoramic images) contains contributions from stellar sources also. In the next subsection, we estimate the contribution of point sources, which turns out to be a negligible fraction of the total emission detected.

\subsubsection{Contribution of point sources to the MSX data}

The panoramic images from MSX includes the contribution from the point sources, some of which have been detected and are catalogued in the MSX PSC. The sources from our MSX PSC sample contribute nearly $4.5 \%, 1.9 \%, 3.5 \%$ and $2.9 \%$ of the total mid infrared emission in the 8, 12, 14 and $21 \mu \mathrm{m}$ bands, respectively. (The corresponding fractions considering all the MSX PSC sources are: $4.7 \%, 1.9 \%, 3.8 \%$ and $3.1 \%$.) However, these correspond only to the sources brighter than the completeness limit of the catalog. In other words, there are (1) unresolved sources (MSX angular resolution is $\sim 18^{\prime \prime}$ ) and (2) sources fainter than the sensitivity limit, contributing to the intensity in these images, apart from the cataloged point sources. To estimate the contribution of unresolved and faint stellar sources in the mid infrared MSX data, we have used the modified Besançon model described earlier (Sect. 3.1.1). The contributions from stars in the selected latitude range for various longitude zones have been determined from the model simulations. Integrated fluxes at $7 \mu \mathrm{m}$ from all stars within a heliocentric distance of $20 \mathrm{kpc}$ and apparent brightness higher than $m_{\text {lim }}\left([7]<m_{\text {lim }}\right.$, where $\left.m_{\text {lim }}=6.5,7.0, \ldots ., 14.0\right)$, have been determined. It was noted that beyond $m_{\mathrm{lim}}=12$, the increase in the value of the integrated flux was insignificant $(\sim 2 \%)$. The ratio of integrated fluxes for sources with [7] $<14.0$ (representing the entire Galaxy) and [7] $<6.5$ (MSX PSC completeness at $A$ band; Sect. 3.1.1) is found to be $\sim$ 1.4. Carrying out similar model simulations at $15 \mu \mathrm{m}$ and considering the completeness of MSX PSC at $D$ band, we find the ratio of corresponding integrated fluxes is $\sim 1.3$. It may be noted that the values of these ratios are in fact upper limits, since the interstellar extinction 

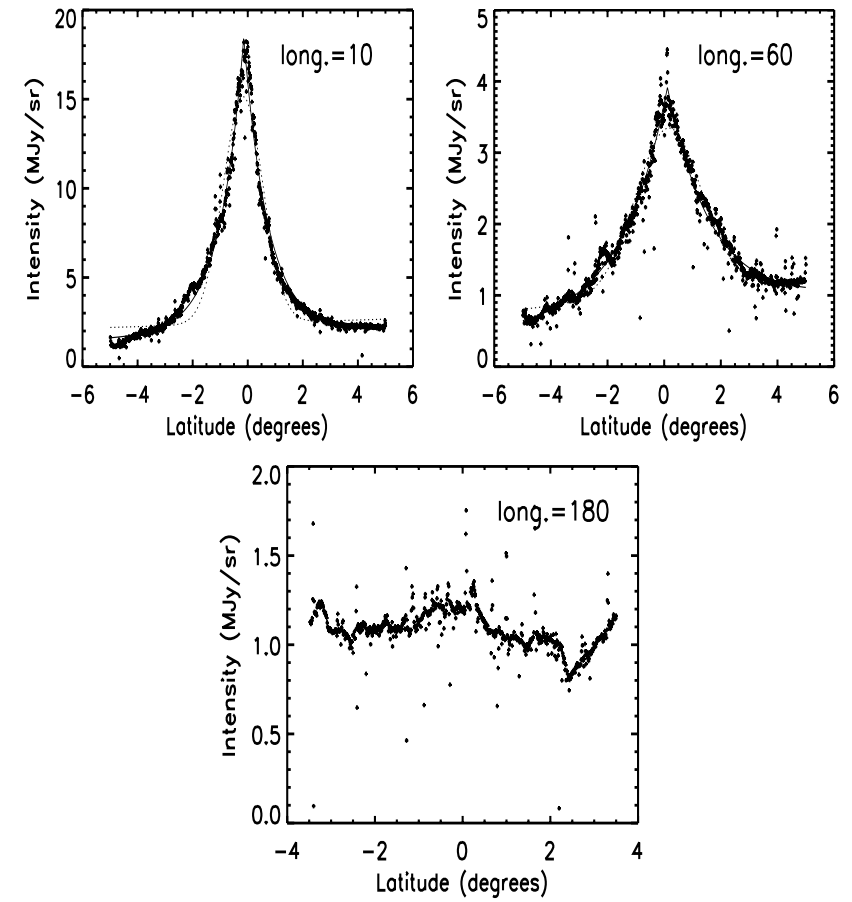

Fig. 4. Latitude profiles of MSX A band intensity for longitude bins $10^{\circ}, 60^{\circ}$ and $180^{\circ}$. The solid and the dotted lines represent best fitting exponential and Gaussian functions, respectively (for $l=10^{\circ}$ and $60^{\circ}$ ).

has been neglected in the model simulations. The above implies that, the contribution of all stars towards the total emission is $\sim 6.3 \%$ and $\sim 4.6 \%$ for 8 and $14 \mu \mathrm{m}$ MSX bands respectively. If we adopt a value of 1.4 for the ratio of integrated fluxes for the 12 and $21 \mu \mathrm{m}$ bands also, then the stellar contributions in the respective bands would be $\sim 2.7 \%$ and $\sim 4.1 \%$. Therefore, we conclude that most of the total mid infrared emission detected in the MSX bands are of diffuse origin. From a study of the diffuse infrared emission (for $|b|>10^{\circ}$ ) of our Galaxy using the IRAS data, Boulanger \& Pérault (1988) found that the point sources account for $10 \% \pm 2 \%$ of the $12 \mu \mathrm{m}$ flux.

\subsubsection{Latitude distribution of diffuse emission and warp signature from MSX data}

The latitude distribution of diffuse emission in the 4 MSX bands, has been studied following an analysis similar to that used for the stars (Sect. 3.1.2), but for $|b| \leq 5^{\circ}$. Exponential and Gaussian functions (see Eqs. (1) and (2)) have been fitted to the latitude profiles of intensity at respective bands. The fits are found to be good for the inner Galaxy $\left(-90^{\circ}<l \leq 90^{\circ}\right)$ but very poor towards the outer Galaxy, similar to the stellar case. The latitude profiles of intensity in A band for three representative longitude bins $\left(10^{\circ}, 60^{\circ}\right.$ and $180^{\circ}$ ) have been presented in Fig. 4, which also displays the fitted functions for the two bins in the inner Galaxy. Again, we find that the exponential provides better fits than the Gaussian for all the cases, as expected. Henceforth, we discuss the exponential only. The average values (and standard deviation) of the angular scale height parameter, $\gamma_{\mathrm{e}}$, from
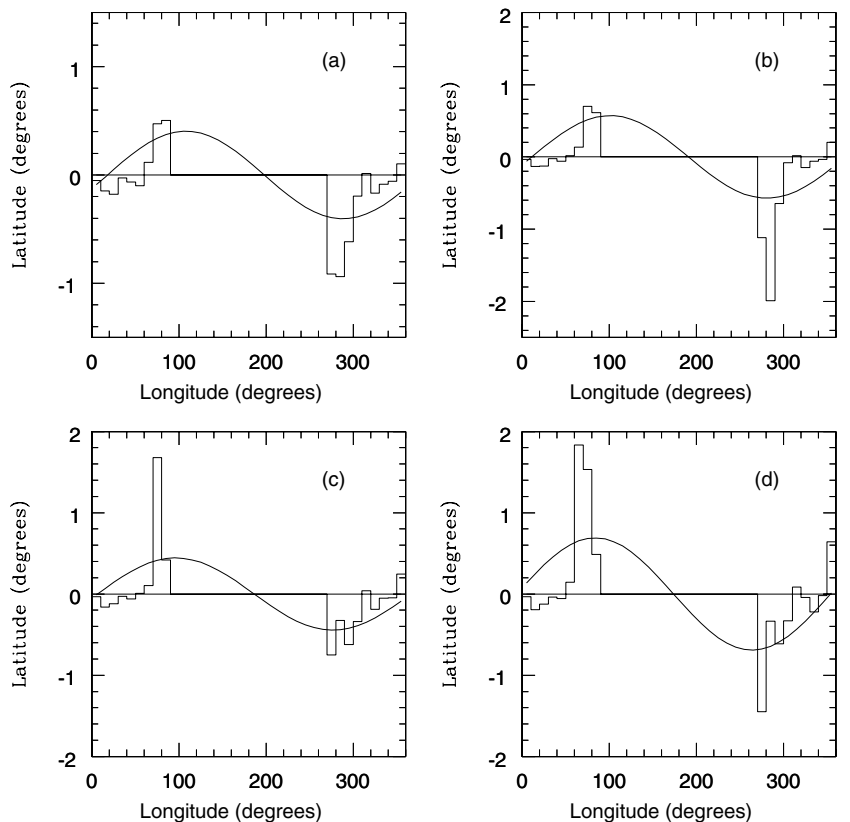

Fig. 5. The variation of the location of Galactic midplane with longitude as obtained by fitting exponential to the latitude profile (LP) of the total (diffuse) emission measured in 4 MSX bands (histogram) for the inner Galaxy. The solid lines refer to the extracted WS in a) $8 \mu \mathrm{m}$, b) $12 \mu \mathrm{m}$, c) $14 \mu \mathrm{m}$, and d) $21 \mu \mathrm{m}$ bands.

the 18 longitude bins, have been listed in Table 1. It may be commented here that, the inferred $\gamma_{\mathrm{e}}$ should be immune to any residuals from the zodiacal subtraction, because: (i) the angular scale of zody emission is much larger than typical $\gamma_{\mathrm{e}}$; and (ii) the background terms (constant and linear in $b$ ) should absorb all components varying slowly with $b$.

In order to search for WS in the diffuse emission, we fitted sinusoids to the locations of profile peak at various longitudes, $\beta_{\mathrm{e}}^{\mathrm{diff}}(l)$, for all 4 MSX bands (similar to the stellar case; Eq. (3)). Once again, the WS has been detected. The best fit values of the parameters of the WS, viz., amplitude and phase are listed in Table 2 . The $\beta_{\mathrm{e}}^{\text {diff }}(l) \mathrm{s}$ and corresponding best fitting sinusoids are presented in Fig. 5 .

We have also pursued the statistical approach (SI; similar to the stellar case) to extend the search for WS over the entire Galactic longitude range. Two measures of central tendencies have been defined for the latitude distribution of intensity at various $l$. The intensity weighted central latitude $B_{\text {intwt }}(l)$ of the diffuse emission is defined by:

$B_{\text {intwt }}(l)=\sum_{b_{i}=-5^{\circ}}^{5^{\circ}} b_{i} f_{i} / \sum_{b_{i}=-5^{\circ}}^{5^{\circ}} f_{i}$

where $b_{i}$ and $f_{i}$ are the latitude and the diffuse emission intensity for the pixel $i$, considering all pixels within the particular longitude bin $l$. The median latitude, $B_{\text {med }}(l)$, is defined to be that latitude, on both sides of which the areas under the intensity vs. latitude curve are equal,

$\sum_{b_{i}=-5^{\circ}}^{B_{\text {med }}(l)} f_{i} \Delta b=\sum_{b_{j}>B_{\operatorname{med}}(l)}^{5^{\circ}} f_{j} \Delta b$ 

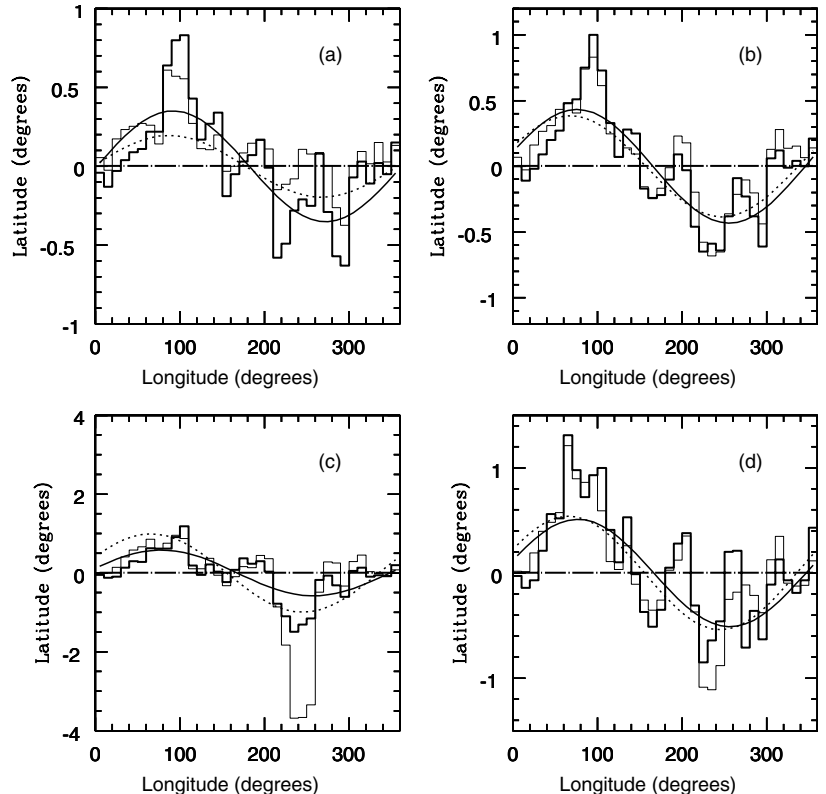

Fig. 6. The variation of the SIs of the Galactic midplane, viz. intensity weighted mean $\left(B_{\text {intwt }}\right.$; thick line histogram) and median $\left(B_{\text {med }}\right.$; thin line histogram) latitudes as a function of longitude for the diffuse emission in the 4 MSX bands: a) $8 \mu \mathrm{m}$, b) $12 \mu \mathrm{m}$, c) $14 \mu \mathrm{m}$, and d) $21 \mu \mathrm{m}$. The solid and the dotted curves refer to the extracted WSs for the intensity weighted and median latitudes, respectively.

where $\Delta b$ is the pixel size along latitude (36"). This Eq. (5) is solved to determine $B_{\text {med }}$ for each of the 36 longitude bins. The statistical indicators (SI) for the Galactic midplane, $B_{\text {intwt }}$ and $B_{\text {med }}$, as a function of Galactic longitude for each MSX band are presented in Fig. 6. These SIs for diffuse emission have also been fitted with sinusoids to search for WS.

Table 3 lists the best fit parameters corresponding to the extracted WS for the SI. We observe that even in this case of diffuse emission from warm interstellar dust, there is a clear warp-like signature in all the four bands. Interestingly, the values of the phases are very similar to the stellar case. For both the SIs we find that $\mathrm{C}$ and $\mathrm{E}$ bands show similar amplitudes within errors. However, for the D band we find that the $B_{\text {med }}$ shows a larger amplitude of WS than $B_{\text {intwt }}$ and vice-versa for the A band. This is in contrast to the stellar case where the median showed a larger amplitude of WS in all the 4 bands. For the case of SI of central tendencies, a better quality of fit to sinusoid is obtained for $B_{\text {med }}$ than $B_{\text {intwt }}$, for almost all the cases.

\subsection{Latitude distribution of emission and warp signature from DIRBE data}

We have searched for WS from the emission of the Galactic plane over the entire infrared waveband using the DIRBE images following an analysis identical to that for MSX images (Sect. 3.2.2). Despite poorer angular resolution, the large number of DIRBE wavebands (10) covering near $(1.25 \mu \mathrm{m})$ to far $(240 \mu \mathrm{m})$ infrared makes it a good candidate for comparison. The extracted characteristic angular scales obtained from fitting latitude profiles (LP) with exponential functions, $\gamma_{\mathrm{e}}$, for the inner Galaxy have been listed in Table 1. The SIs for the Galactic
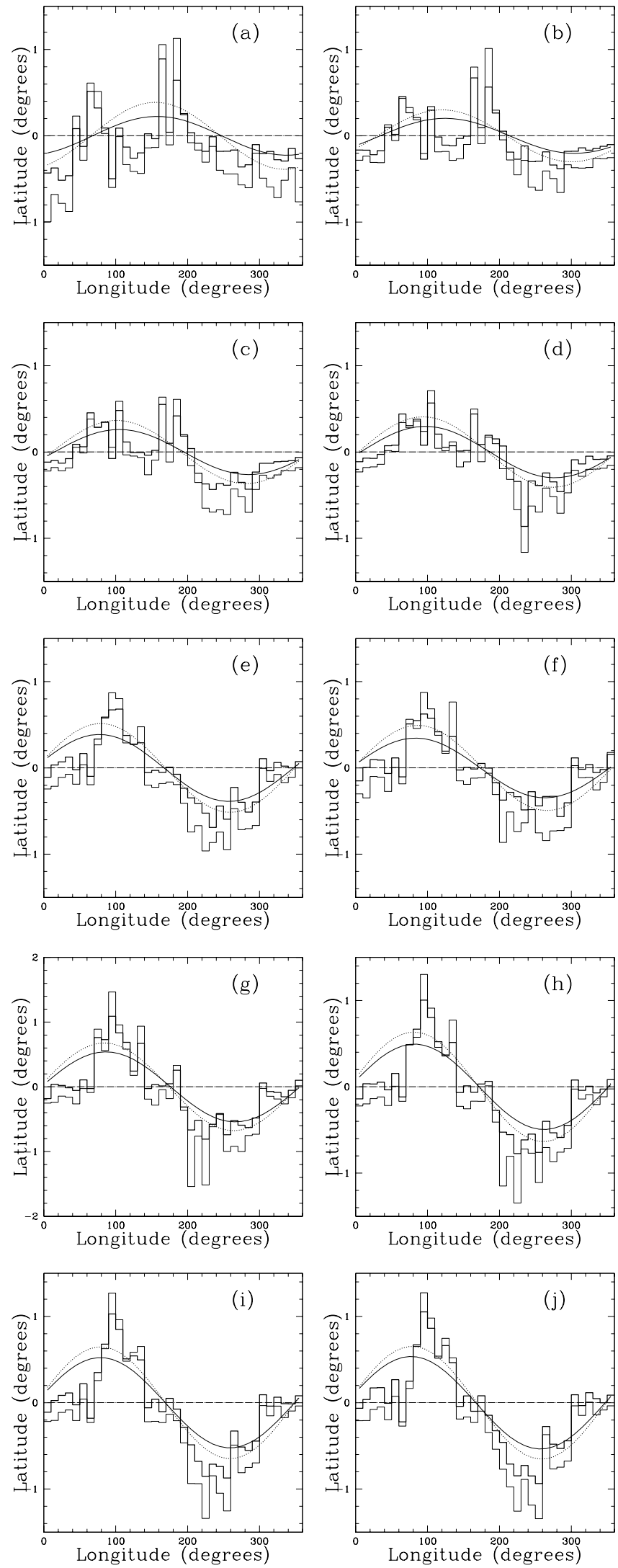

Fig. 7. Same as in Fig. 6 but for DIRBE intensity data in the ten bands: a) $1.25 \mu \mathrm{m}$, b) $2.2 \mu \mathrm{m}$, c) $3.5 \mu \mathrm{m}$, d) $4.9 \mu \mathrm{m}$, e) $12 \mu \mathrm{m}$, f) $25 \mu \mathrm{m}$, g) $60 \mu \mathrm{m}$, h) $100 \mu \mathrm{m}$, i) $140 \mu \mathrm{m}$ and j) $240 \mu \mathrm{m}$ band. The solid and the dotted lines refer to the extracted warp signatures corresponding to the best fit sinusoid to $B_{\text {intwt }} \mathrm{s}$ and $B_{\text {med }} \mathrm{s}$, respectively. 
midplane, $B_{\text {intwt }}$ and $B_{\text {med }}$ for the entire longitude range, for each DIRBE band have been presented in Fig. 7. These SIs have also been searched for WS and the resulting best fitting sinusoids have been overplotted on the same figure.

The WS has been detected in all the three (one LP and two SI) cases in each of the DIRBE bands. Even the phases of the WS, as evident from Table 2 (LP) (and 3; SI), obtained for 9 (8) of the 10 bands are consistent with those obtained from MSX data for the stars as well as the diffuse mid infrared emission. It may be noted that at $1.25 \mu \mathrm{m}$, the only band where the WS phase (and also the amplitude) deviates significantly from the other bands, the errors are the largest. This could be because the emission at $1.25 \mu \mathrm{m}$ originates mainly from the stars, which would be affected most by extinction due to interstellar dust.

\section{Characteristic angular and linear scale heights}

In the present study, we have determined the characteristic angular scales of emission along the Galactic latitude, which need to be translated to linear scales for drawing physical inferences. For this purpose, we have carried out calculations based on geometric description of the Galactic disk. The Galactic disk has been considered to be of radius $14 \mathrm{kpc}$ and thickness $700 \mathrm{pc}$, with $R_{\odot}=8 \mathrm{kpc}$. Our description neglects the effects of the Galactic bulge, the halo as well as any warp feature. The effect of these simplifications on our results is expected to be negligible.

We need to estimate the integrated emission as a function of Galactic latitude for a given longitude, $I(b)$, with linear scale height $\left(z_{\mathrm{h}}\right)$ and radial scale length $\left(R_{\mathrm{l}}\right)$ as input parameters. It is reasonable to expect the observable intensity to scale with the integral of the emission along the line of sight, $\rho$ (Hammersley et al. 1995). Hence, we write:

$I(b) \propto \int_{0}^{\rho_{\max }(l, b)} \mathrm{e}^{-\frac{\rho|\sin (b)|}{z_{\mathrm{h}}}-\frac{R}{R_{1}}} \mathrm{~d} \rho$

for a given $l$, where $R=\sqrt{R_{\odot}^{2}+(\rho \cos b)^{2}-2 R_{\odot} \rho \cos b \cos l}$ is the galactocentric distance and $\rho_{\max }(l, b)$ is the maximum length along a given line of sight which is only constrained by the finite size of the disk. These synthetic latitude profiles are found to be better represented by exponential functions than Gaussian as expected. We have fitted exponential functions of the form $\rho_{o} \mathrm{e}^{-\frac{|b|}{\psi_{\mathrm{h}}}}+\eta$ to these latitude profiles, with $\rho_{o}, \psi_{\mathrm{h}}$ and $\eta$ as free parameters. The $\psi_{\mathrm{h}}$ corresponds to characteristic angular scale and $\eta$ represents the background (no latitude dependent background is anticipated due to the symmetry of the geometry). The study has been carried out at two representative longitudes $30^{\circ}$ and $90^{\circ}$. The lines of sight closer towards the Galactic centre have been avoided due to difficulty in comparing the calculations with measurements, since our description neglects the more complex details (like the bulge) which could be important. The following values of $R_{1}: 1.5,2.0,2.5, \ldots, 7.0$, 7.5 and $\infty \mathrm{kpc}\left(R_{1}=\infty\right.$ corresponds to constant radial density distribution) have been explored. The set of $z_{\mathrm{h}}$ values explored are $40,50,60, \ldots, 90,100,125,150, \ldots, 275,300$ pc.
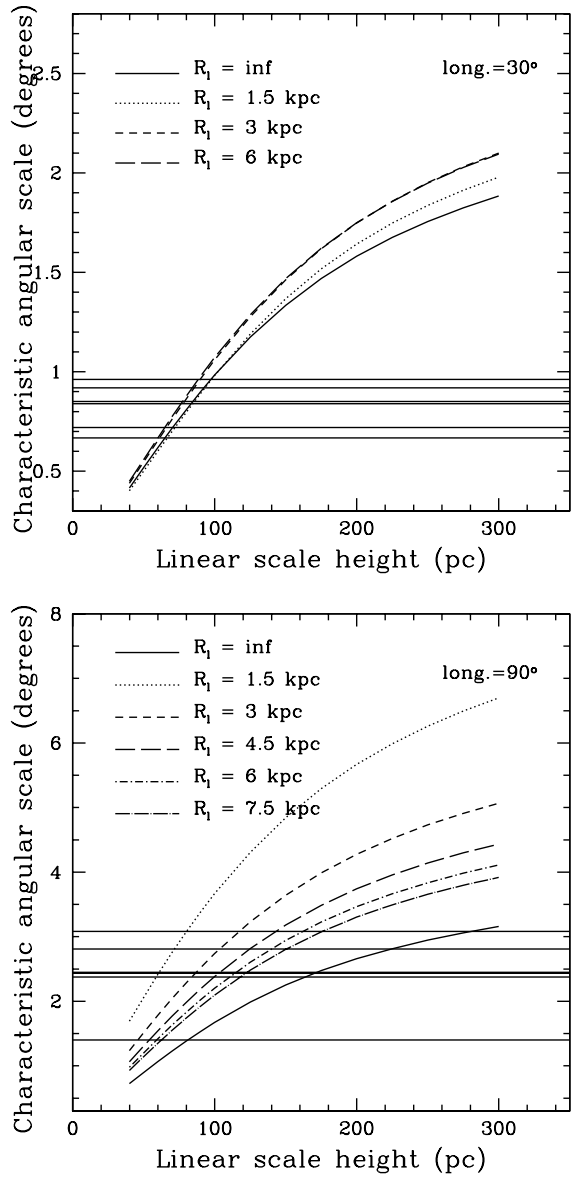

Fig. 8. Relations between the linear $\left(z_{\mathrm{h}}\right)$ and the angular $\left(\gamma_{\mathrm{e}}\right)$ scale heights from a geometric description of the Galactic disk (for a heliocentric observer), for different assumed radial scale lengths, $R_{\mathrm{l}}$. The two panels represent longitudes $l=30^{\circ}$ and $l=90^{\circ}$. The $\gamma_{\mathrm{e}} \mathrm{s}$ obtained from the exponential fits for the diffuse mid infrared emission in 4 MSX bands (for A, C, D and E bands: $0.85^{\circ}, 0.92^{\circ}, 0.96^{\circ}$ and $0.67^{\circ}$ for $l=30^{\circ}$; and $3.08^{\circ}, 2.45^{\circ}, 2.43^{\circ}$ and $2.37^{\circ}$ for $l=90^{\circ}$ ) and the two DIRBE bands (for 12 and $25 \mu \mathrm{m}$ bands: $0.84^{\circ}, 0.72^{\circ}$ for $l=30^{\circ}$; and $2.81^{\circ}, 1.40^{\circ}$ for $l=90^{\circ}$ ) are also plotted as horizontal solid lines for comparison.

The results of our calculations (for $l=30^{\circ}$ and $90^{\circ}$ ) connecting the characteristic angular scale $\left(\gamma_{\mathrm{e}}\right)$ with linear scale height $\left(z_{\mathrm{h}}\right)$, for selected values of the radial scale lengths $\left(R_{1}\right)$, have been presented in Fig. 8. The larger separation among curves corresponding to different values of $R_{1}$ for $l=90^{\circ}$ as compared to $l=30^{\circ}$, is entirely expected from the shape and size of the Galactic disk and the heliocentric location of the observer. Overplotted as horizontal lines in this figure, are the values of $\gamma_{\mathrm{e}}$ for mid infrared wavebands at corresponding longitudes, as obtained earlier from fitting the observed latitude profiles (Sects. 3.2.2 and 3.3) for the 4 MSX bands and 2 DIRBE bands (12 and $25 \mu \mathrm{m}$ ). This comparison helps constraining the values of $R_{1}$ and $z_{\mathrm{h}}$ for the distribution of warm interstellar dust. From the overlap of the observed and the model curves for $l=30^{\circ}$, we infer that the preferred range of $z_{\mathrm{h}}$, is $\sim 60-100 \mathrm{pc}$, while $R_{1}$ cannot be constrained due to the 


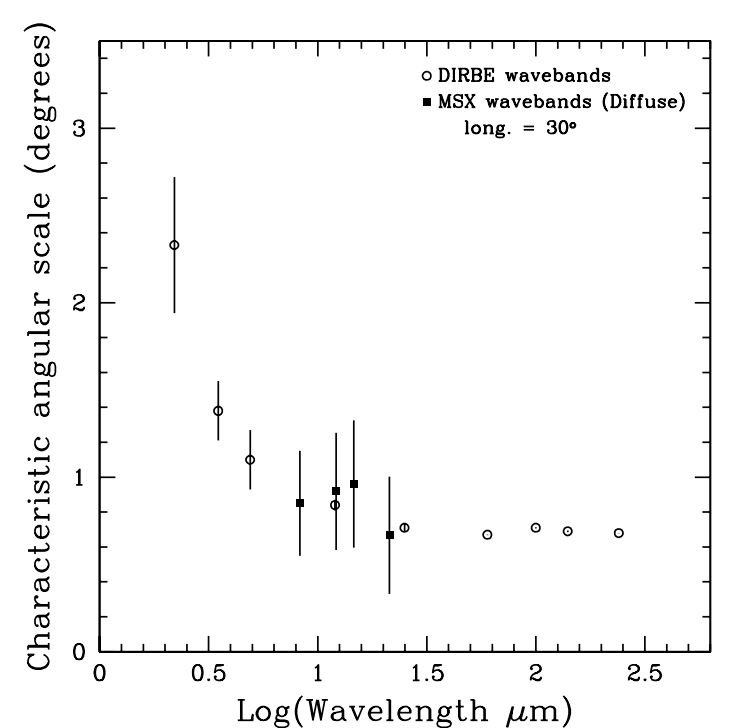

Fig. 9. Wavelength dependence of the angular scale height, $\gamma_{\mathrm{e}}$, for the longitude bin $l=30^{\circ}$. The open circles represent the DIRBE wavebands for $\lambda \geq 2.2 \mu \mathrm{m}$ and the solid squares represent the diffuse emission in the MSX bands at 8,12, 14 and $21 \mu \mathrm{m}$. The error bars indicate $1 \sigma$ uncertainty. At longer wavelengths the errors are smaller than the symbol size.

insensitivity to this parameter. However, the situation at $l=90^{\circ}$ is quite favourable and provides a handle for $R_{1}$. Assuming $z_{\mathrm{h}}$ to be in the above range, it is evident that the radial scale length, $R_{1}$, should be less than $\sim 6 \mathrm{kpc}$. Although the value of $\gamma_{\mathrm{e}}$ obtained from DIRBE $25 \mu \mathrm{m}$ band, $1.4^{\circ}$, seems incompatible with the above (Fig. $8, l=90^{\circ}$ ), we note that the values corresponding to the immediate neighbouring longitude bins are systematically higher $\left(2.0^{\circ}\right.$ and $\left.2.2^{\circ}\right)$, consistent with the above constraint on $R_{1}$. Giving equal weightage to the six mid-IR bands, we find the most favoured value of $R_{\mathrm{l}}$ to be $2.81 \pm 0.63 \mathrm{kpc}$ corresponding to $z_{\mathrm{h}}=80 \mathrm{pc}$ (central value of our implied range). Similarly, for the far infrared bands representing the cold interstellar dust, the scale heights $z_{\mathrm{h}}$ have been found to be $\sim 60 \mathrm{pc}$.

In addition, it is instructive to compare the characteristic angular scales, $\gamma_{\mathrm{e}}$, obtained in the present study as a function of wavelength. For this, we have selected a moderate longitude bin $\left(l=30^{\circ}\right)$ where the complexities due to additional components of the Galactic structure near the Galactic center is absent, yet the signal to noise ratio is reasonably good for reliable determination of $\gamma_{\mathrm{e}} \mathrm{s}$. The variation of $\gamma_{\mathrm{e}}$ with wavelength, at $l=30^{\circ}$, corresponding to the DIRBE and MSX wavebands $(\lambda \geq 2.2 \mu \mathrm{m})$ have been presented in Fig. 9. We find that $\gamma_{\mathrm{e}}$ drops with wavelength from near to mid infrared and remains more or less constant (within errors) thereafter upto far infrared wavelengths.

While the near infrared emission is expected to be dominated by stars, the mid infrared bands sample the warm interstellar dust in the vicinity of star forming regions. On the other hand, the far infrared emission is predominantly from cold dust heated by average interstellar radiation field. The observed decrease in $\gamma_{\mathrm{e}}$ obtained from 2.2 to $8.3 \mu \mathrm{m}$ bands could possibly be linked to the extinction due to dust. Typical wavelength dependence of dust extinction being $\propto \lambda^{-\alpha}(1<\alpha<2)$, the extinction is expected to be much larger for the near infrared as compared to mid or far infrared wavebands. The effect of extinction is largest on the Galactic plane $(b=0)$ and progressively reduces at higher latitudes. The observed peak emission being reduced due to attenuation, the fit to the data could indicate an effectively larger angular scale height.

\section{Comparison with other results}

In this section we compare our results about the structure and WS of the Galactic disk with other studies of the distribution of stellar and the interstellar components.

\subsection{Indicators of structure of the Galactic disk}

The present study has constrained the scale height and radial scale length for the distribution of warm interstellar dust $\left(z_{\mathrm{h}}=\right.$ 60-100 pc, $R_{1}<6 \mathrm{kpc}$; Sect. 4). Our $z_{\mathrm{h}}$ is close to that for the ISM (140 pc) from the successful model of the Galactic structure by Robin et al. (2003), although their $R_{1}$ is higher $(4.5 \mathrm{kpc})$. The distribution of dust studied by Freudenreich (1998) using near infrared measurements imply: $z_{\mathrm{h}} \sim 150-200 \mathrm{pc}$ and $R_{1} \sim 3.0-3.3 \mathrm{kpc}$, which is in reasonable agreement with our $R_{1}$. Drimmel \& Spergel (2001), have obtained the values $z_{\mathrm{h}}=134 \mathrm{pc}$ and $R_{1}=2.26 \mathrm{kpc}$ for the cold dust component, using DIRBE $240 \mu \mathrm{m}$ data, which compares very well with our results.

\subsection{Signatures of Galactic warp}

In this subsection, our results of WS have been compared with other studies for the stellar, the warm and cold interstellar dust components. Table 4 presents the summary of this overall comparison.

\subsubsection{Warp signature from the stellar component}

The WS for the stellar component obtained here using the MSX PSC, is in very good agreement with that obtained by Djorgovski \& Sosin (1989) from the distribution of IRAS point sources, despite their sample covering a much larger latitude range of $|b| \leq 10^{\circ}$. The estimates for the WS phase, $\phi$, obtained here from both the approaches (using latitude profiles for the inner Galactic plane, LP; and using statistical indicators for the entire Galactic plane, SI) match extremely well with the IRAS results (see Table 4). The WS amplitude obtained for the SI case is also consistent with the IRAS result, although the LP case (representing only the inner Galaxy) implies somewhat larger value. Similar consistency is also observed for the results obtained here using DIRBE data in $L(3.5 \mu \mathrm{m})$ and $M(4.9 \mu \mathrm{m})$ bands for both LP and SI. Lopez-Corredoira et al. (2002b) have analysed the data from Two Micron All Sky Survey (2MASS) in the context of Galactic warp. Their best fit warped model (Fig. 15, $b=+3^{\circ} / b=-3^{\circ}$ ) corresponds to a value of $+2^{\circ}$ for our phase parameter $\phi$, which is again similar to those obtained here from all the four bands of MSX PSC as well as $L$ and $M$ bands of DIRBE. Freudenreich (1998) from a study of the 
Table 4. Comparison of parameters of warp signature (amplitude and phase) for the Galactic disk obtained from different studies.

\begin{tabular}{|c|c|c|c|c|c|}
\hline $\begin{array}{l}\lambda(\mu \mathrm{m}) \\
\text { (band) }\end{array}$ & Survey & Comp. & $\overline{\mathrm{LP} / \mathrm{SI}^{a}}$ & $\begin{array}{c}\text { WS amplitude } \\
\text { (deg) }\end{array}$ & $\begin{array}{c}\text { WS phase } \\
\text { (deg) }\end{array}$ \\
\hline $\begin{array}{c}2.2^{b} \\
(\mathrm{NIR})\end{array}$ & 2MASS & Stellar & $\ldots$ & $\ldots$ & 2 \\
\hline $\begin{array}{c}2.2^{c} \\
(\mathrm{NIR})\end{array}$ & DIRBE & Stellar & $\begin{array}{l}\text { LP } \\
\text { SI }\end{array}$ & $\begin{array}{c}0.5 \\
0.2-0.3\end{array}$ & $\begin{array}{c}19 \\
30-34\end{array}$ \\
\hline $\begin{array}{c}3.5,4.9^{c} \\
(\mathrm{NIR})\end{array}$ & DIRBE & Stellar & $\begin{array}{l}\text { LP } \\
\text { SI }\end{array}$ & $\begin{array}{l}0.3-0.4 \\
0.3-0.4\end{array}$ & $\begin{array}{c}15 \\
4-15\end{array}$ \\
\hline $\begin{array}{c}8.3-21^{c} \\
(\mathrm{MIR})\end{array}$ & MSX & Stellar & $\begin{array}{l}\text { LP } \\
\text { SI }\end{array}$ & $\begin{array}{l}0.4-0.5 \\
0.2-0.4\end{array}$ & $\begin{array}{c}3-7 \\
1-18\end{array}$ \\
\hline $\begin{array}{c}12-60^{d} \\
(\mathrm{MIR} / \mathrm{FIR})\end{array}$ & IRAS & Stellar & $\ldots$ & $0.2-0.3$ & $0-10$ \\
\hline $\begin{array}{c}8.3^{c} \\
(\mathrm{MIR})\end{array}$ & MSX & Warm dust & $\begin{array}{l}\text { LP } \\
\text { SI }\end{array}$ & $\begin{array}{c}0.4 \\
0.2-0.4\end{array}$ & $\begin{array}{c}18 \\
-3-2\end{array}$ \\
\hline $\begin{array}{l}12-21^{c} \\
(\mathrm{MIR})\end{array}$ & MSX & Warm dust & $\begin{array}{l}\text { LP } \\
\text { SI }\end{array}$ & $\begin{array}{c}0.4-0.7 \\
0.4-1\end{array}$ & $\begin{array}{c}-6-11 \\
-25--12\end{array}$ \\
\hline $\begin{array}{l}12,25^{c} \\
(\mathrm{MIR})\end{array}$ & DIRBE & Warm dust & $\begin{array}{l}\text { LP } \\
\text { SI }\end{array}$ & $\begin{array}{c}0.4 \\
0.4-0.5\end{array}$ & $\begin{array}{c}11-18 \\
-13--4\end{array}$ \\
\hline $\begin{array}{c}60-240^{c} \\
\text { (FIR) }\end{array}$ & DIRBE & Cold dust & $\begin{array}{l}\text { LP } \\
\text { SI }\end{array}$ & $\begin{array}{l}0.4-0.5 \\
0.5-0.7\end{array}$ & $\begin{array}{c}12-16 \\
-13--4\end{array}$ \\
\hline
\end{tabular}

${ }^{a}$ From Latitude Profiles, $-90^{\circ}<l \leq 90^{\circ}$ (LP). / Statistical Indicators, $0^{\circ}<l \leq$ $360^{\circ}$ (SI).

${ }^{b}$ Lopez-Corredoira et al. (2002b).

${ }^{c}$ Present work.

${ }^{d}$ Djorgovski \& Sosin (1989).

Galactic disk obtain $-0.08^{\circ}<\phi<0.8^{\circ}$, which again is consistent with our results.

It is interesting to note that, stellar distributions obtained from MSX and IRAS show fair amount of symmetry between the positive and negative peaks of the warp signature, unlike the case for H I distribution (Burton 1988).

\subsubsection{Warp signature from the interstellar dust component}

The diffuse mid infrared (8-21 $\mu \mathrm{m})$ emission detected by MSX is expected to originate from the warmer $(T \gtrsim 100 \mathrm{~K})$ component of the interstellar dust. In order to be heated to such high temperatures, the dust grains need strong local radiation fields, e.g. near young stellar objects (OB stars). Hence, detection of WS from diffuse mid infrared emission provides information about the distribution of warm dust around luminous young stars in the Galactic plane. Our results of the WS for the diffuse component obtained from the 4 bands of MSX and the two mid infrared bands of DIRBE (12 and $25 \mu \mathrm{m}$ ) have also been compared in Table 4 . The $\phi$ s obtained are consistent within errors. The amplitudes inferred from the MSX and DIRBE are similar with the former being slightly larger. An identical systematic shift $\left(\sim 20^{\circ}\right)$ in $\phi$ can be noticed between the values obtained for the inner Galactic plane (from LP) and those for the entire disk (from SI), for all the mid infrared bands of MSX as well as DIRBE.

The far infrared emission originates from the colder ( $T \lesssim 30 \mathrm{~K}$ ) component of interstellar dust in the Galactic plane. The WS detected in 60-240 $\mu$ m wavebands of DIRBE should correspond to the distribution of this component. The $\phi$ s agree very well with those for the stellar as well as the warm dust component (Table 4). The amplitude of the WS is similar to that obtained for the warmer dust.

\section{Summary}

Taking advantage of the recent high resolution $\left(\sim 18^{\prime \prime}\right)$ Galactic plane survey in the mid infrared $(8,12,14$ and $21 \mu \mathrm{m})$ by the Midcourse Space Experiment (MSX), the distribution of stars (from the Point Source Catalog) as well as warm interstellar dust (from the panoramic images) in the Galactic disk have been investigated. The location of the Galactic mid-plane and the characteristic angular scales along latitude have been obtained from latitude profiles $\left(-90^{\circ}<l \leq 90^{\circ}\right)$, and statistical 
indicators (entire Galactic plane). Using a simple geometric description of the Galactic disk, the angular scales have been translated to scale heights $\left(z_{\mathrm{h}}\right)$ and radial length scales $\left(R_{1}\right)$. The following constraints have been obtained for the distribution of warm $(T \gtrsim 100 \mathrm{~K})$ interstellar dust in the Galactic disk: $R_{1}<6 \mathrm{kpc}$ and $60 \lesssim z_{\mathrm{h}} \lesssim 100 \mathrm{pc}$. These are consistent with results from other studies.

Signatures of warp-like feature in the distribution of stars as well as warm interstellar dust have been searched for using the inferred locations of the Galactic mid-plane as a function of longitude. The warp signature (WS) has been detected in all the cases: for point sources as well as diffuse emission in each of the 4 MSX bands. The WS for each case has been quantified by two parameters depicting its amplitude and phase (longitude of the line of nodes).

Our analysis/study of the mid infrared emission has been extended to cover the entire infrared waveband by using the data from the DIRBE/COBE experiment (10 bands; $1.25-240 \mu \mathrm{m})$. Despite limited angular resolution of DIRBE, signatures of warp have been detected in each band with very similar phase as found from the MSX data. The inferred amplitudes of WS lie in the range: $0.2^{\circ}-0.7^{\circ}$ (for $\lambda \geq 2.2 \mu \mathrm{m}$ ) in latitude. Results from the present study have also been compared with those from other similar studies based on the IRAS and 2MASS surveys.

Acknowledgements. It is a pleasure to thank the referees - M. Lopez-Corredoira and the anonymous referee - for comments and suggestions, which greatly improved the scientific content of the paper. We thank Sean Carey and Michael Egan for providing us with MSX panoramic mosaics. This research made use of data products from the Midcourse Space Experiment, the processing of which was funded by the Ballistic Missile Defense Organization with additional support from NASA Office of Space Science. This research has also made use of the NASA/IPAC Infrared Science Archive, which is operated by the Jet Propulsion Laboratory, California Institute of Technology, under contract with National Aeronautics and Space Administration.

The COBE datasets were developed by the NASA Goddard Space Flight Center under the guidance of the COBE Science Working Group. We acknowledge the use of the Legacy Archive for Microwave Background Data Analysis (LAMBDA). Support for LAMBDA is provided by the NASA Office of Space Science. We thank Annie Robin for letting us use her model of stellar population synthesis and for helpful discussions. S.V. acknowledges the TIFR Endowment Fund for partial financial support.

\section{References}

Battaner, E., Florido, E., \& Sanchez-Saavedra, M. L. 1990, A\&A, 236, 1

Battaner, E., Garrido, J. L., Sanchez-Saavedra, M. L., \& Florido, E. 1991, A\&A, 251, 402

Beichman, C. A., Neugebauer, G., Habing, H. J., Clegg, P. E., \& Chester, T. J. 1988, IRAS Catalogs \& Atlases, Volume 1: Explanatory Supplement

Binney, J. 1978, MNRAS, 183, 779

Binney, J. 1992, ARA\&A, 30, 51

Boggess, N. W., Mather, J. C., Weiss, R., et al. 1992, ApJ, 397, 420

Boulanger, F., \& Pérault, M. 1988, ApJ, 330, 964

Burke, B. F. 1957, AJ, 62, 90

Burton, W. B. 1988, Galactic and Extragalactic Radio Astronomy, ed. G. L. Verschuur, \& K. I. Kellerman (Berlin: Springer-Verlag)

Debattista, V. P., \& Sellwood, J. A. 1999, ApJ, 513, L107

Djorgovski, S., \& Sosin, C. 1989, ApJ, 341, L13

Drimmel, R., \& Spergel, D. N. 2001, ApJ, 556, 181

Egan, M. P., Price, S. D., Moshir, M. M., Cohen, M., \& Tedesco, E. 1999, MSX Point Source Catalog version 1.2 Explanatory Guide, AFRL-VS-TR-1999-1522, (Springfield: NTIS)

Freudenreich, H. T. 1996, ApJ, 468, 663

Freudenreich, H. T. 1998, ApJ, 492, 495

Freudenreich, H. T., Berriman, G. B., Dwek, E., et al. 1994, ApJ, 429, L69

Hammersley, P. L., Garzon, F., Mahoney, T., \& Calbet, X. 1995, MNRAS, 273, 206

Hunter, C., \& Toomre, A. 1969, ApJ, 155, 747

Jiang, I., \& Binney, J. 1999, MNRAS, 303, L7

Kent, S. M., Dame, T. M., \& Fazio, G. 1991, ApJ, 378, 131

Kennealy, J. P., Noah, P. V., Tedesco, E. F., et al. 1993, CBSD: The Celestial Background Scene Descriptor, PL-TR-93-2215, 5-59, Air Force Research Laborartory

Kerr, F. J. 1957, AJ, 62, 93

Lopez-Corredoira, M., Betancort-Rijo, J., \& Beckman, J. E. 2002a, A\&A, 386, 169

Lopez-Corredoira, M., Cabrera-Lavers, A., Garzon, F., \& Hammersley, P. L. 2002b, A\&A, 394, 883

Lumsden, S. L., Hoare, M. G., Oudmaijer, R. D., \& Richards, D. 2002, MNRAS, 336, 621

Ostriker, E. C., \& Binney, J. J. 1989, MNRAS, 237, 785

Price, S. D., Egan, M. P., Carey, S. J., Mizuno, D. R., \& Kuchar, T. A. 2001, AJ, 121, 2819

Porcel, C., Battaner, E., \& Jimenez-Vicente, J. 1997, A\&A, 322, 103

Robin, A. C., Reylé, C., Derriére, S., \& Picaud, S. 2003, A\&A, 409, 523

Rogstad, D. H., Lockart, I. A., \& Wright, M. C. H. 1974, ApJ, 193, 309 